\title{
Acute renal insufficiency after scorpion sting
}

\author{
G G Ranaweera ${ }^{1}$, V Bavanthan ${ }^{2}$, A L M Nazar ${ }^{2}$, M D S Lokuhetty ${ }^{1}$
}

Ceylon Medical Journal 2015; 60: 31-32

\section{Introduction}

Scorpions are venomous arthropods found worldwide [1]. The sequelae of scorpion stings depend on the composition and amount of venom entering the body. The composition of venom depends on the scorpion species $[1,2]$. Renal insufficiency following a scorpion sting is relatively rare with only 62 reported cases [1].

\section{Case report}

A 53-year old female from Wadduwa, on treatment for hypertension and diabetes for 6 years, sustained a scorpion sting. She developed features of envenomation (vomiting and abdominal pain) within four hours, and haemoglobinuria progressing to anuria suggestive of acute renal insufficiency (ARI)) in the next 24 hours.

She was admitted to hospital on the second day with localized pain, oedema, abdominal pain and anuria. Chest pain and neurological symptoms were absent. She was febrile and blood pressure was $180 / 100 \mathrm{mmHg}$. The total white cell count was $23 \times 10^{3} \mu 1$ with neutrophil leukocytosis, platelet count $88 \times 10^{3} / \mu 1$, C reactive protein $232 \mathrm{mg} / \mathrm{dl}$ (normal $<0.6 \mathrm{mg} / \mathrm{dl}$ ) and serum creatinine 468 $\mu \mathrm{mol} / \mathrm{l}$, and blood picture showed microangiopathic haemolytic anemia. Ultrasound performed on second day of the illness showed that the right and left kidneys were $9.5 \mathrm{~cm}$ and $9.0 \mathrm{~cm}$ in length with preserved corticomedullary demarcation. She was managed conservatively with regular haemodialysis.

Renal biopsy performed in the second week showed haemorrhagic glomerular infarction with acute tubular injury (ATI), and an oedematous interstitium with extravasated red cells and lymphocytes. Two viable glomeruli showed mild mesangial expansion. Capillary thrombosis and fibrinoid necrosis of blood vessels were absent (Figure 1).

She was dependent on dialysis due to persisting renal insufficiency. She defaulted treatment and died four months later.

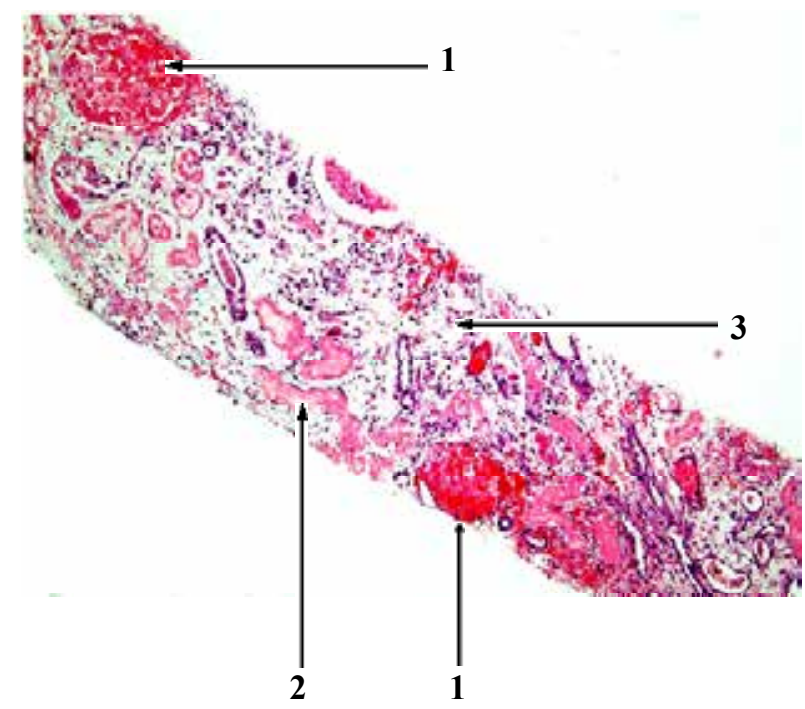

Figure 1. 1. Renal tissue with haemorrhagic infarctions of glomeruli 2.Acute tubular injury, 3. Interstitial oedema and inflammation. (Haematoxyline and Eosine stain $x$ 10)

\section{Discussion}

Around 1500 scorpion species exist, of which only 30 are medically important [1,2]. Of the 16 scorpion species identified from Sri Lanka, family Buthidae is the only known species which causes life threatening systemic effects after envenomation $[2,3]$.

Scorpion venom contains a cocktail of neurotoxins, cardiotoxins, nephrotoxins, hemolytic toxins, acetylcholine esterase, histamine, serotonin and 5-hydroxyptamine [2]. The clinical effects depend on the species and therefore nature of the venom, dose inoculated and other host factors [2]. Envenomation causes overstimulation of adrenergic and cholinergic receptors and an inflammatory response with rapid development of symptoms as witnessed in this patient $[1,2]$. Sixty two cases of scorpion sting nephropathy have been reported from Iran, Tunisia, Turkey, Pakistan and Israel. Implicated mechanisms include direct effect of venom on the kidney and renal damage resulting from intravascular haemolysis, disseminated intravascular

\footnotetext{
${ }^{1}$ Department of Pathology, Faculty of Medicine, University of Colombo and ${ }^{2}$ National Hospital of Sri Lanka, Colombo, Sri Lanka.
}

Correspondence: GGR, e-mail: <gayanimfc@gmail.com>. Received 3 October and revised version accepted 29 December 2014. Competing interests: none declared. 
coagulation (DIC), haemolytic ureamic syndrome (HUS) and hypovolaemia [1]. Renal excretion of concentrated venom results in direct renal tubular damage leading to ATI [4]. Haemoglobin released following intravascular haemolysis also damages renal tubules. Serotonin causes impaired blood flow through the afferent arterioles resulting in ischaemia of the glomeruli [4]. Haemoglobin casts pack the tubules and interfere with the excretory functions, contributing to ARI [4]. Vomiting, cardiac failure and vascular dilatation secondary to inflammatorymediator release also contribute to ATI by hypovolaemia and hypotension $[2,4]$. When there is DIC/HUS microthrombi occlude intra renal blood vessels resulting in thrombotic microangiopathy, with acute ischemic glomerular damage, glomerular capillary endothelial cell swelling, narrowed capillary lumina and mesangial expansion/mesangiolysis. Fibrinoid necrosis, intimal and sub-intimal fibrin deposits, endothelial proliferation and thrombosis of small arteries and arterioles also occur in DIC/HUS [5].

This patient's renal biopsy had extensive glomerular infarction with mesangial hypercellularity of surviving glomeruli, ATI and interstitial changes. Changes secondary to diabetes and hypertension were absent. Direct nephrotoxicity of venom, haemoglobinuria secondary to microvascular haemolysis and hypovolaemia possibly caused ARI in this patient. Additionally thrombotic microangiopathy may have contributed to the ischemic glomerular damage.

Only five of the 62 nephropathy cases documented have had renal biopsies. These have shown mesangial hypercellularity and interstitial nephritis $[1,4]$. None had features of DIC/HUS with ischaemic glomerular damage. All five cases recovered from ARI $[1,4]$. Extensive glomerular infarction most likely caused persistent renal insufficiency in this patient. Having diabetes and hypertension possibly made her kidneys more vulnerable to damage.
Current management of scorpion sting nephropathy includes symptomatic support and treatment with scorpion anti-venom, which is not available in Sri Lanka [2].

Scorpion stings are not uncommon in this country; however life threatening incidences are rare. The species Hottentotta tumulus (Scorpiones: Buthidae) discovery from Jaffna is associated with deaths attributed to acute heart failure following envenomation [3]. This is the first case of acute renal insufficiency reported in South Asia following a scorpion envenomation.

\section{Conclusion}

In Sri Lanka scorpion stings have the potential to cause serious medical complications and death. Therefore healthcare workers should be aware of features of scorpion evenomation and squelae. The general public should be encouraged to seek medical advice following scorpion stings early, especially if symptomatic.

\section{Declaration of Interest}

There are no conflicts of interest.

\section{References}

1. Viswanathan S, Prabhu C. Scorpion sting nephropathy. NDT plus 2011; 4(6): 376-82.

2. Bawaskar HS, Bawaskar PH. Scorpion sting; update. $J$ Assoc Physicians India 2012; 60: 46-55.

3. Ranawana KB, Dinamithra N, Sivansuthan S, Nagasena I I, Kovarik F, Kularatne SAM. First report on Hottentotta tamulus (Scorpiones: Buthidae) from Sri Lanka, and its medical importance. Euscorpius 2013; 155: 1-7.

4. Malhothra KK, Chadha JS. Mirehghan M, Tandon HD. Acute renal failure following scorpion sting. Am J Trop Med Hyg 1978; 27: 623-6

5. Nelson G, Rosai J. Urinary tract. In: Rosai J. Rosai and Ackerman's Surgical Pathology.10th ed. Missouri: Elsevier, 2011: 1101-246. 